\title{
INFLUENCE OF LOG TEMPERATURE IN IRREGULARITIES ON STRAND GEOMETRY DETECTED BY DIGITAL IMAGE ANALYSIS
}

\author{
Victor Gaete-Martinez $z^{1}$,Stephen M. Shaler ${ }^{2}$
}

\begin{abstract}
Oriented Strand Board (OSB) wood strands, while often idealized as being rectangular and slender objects, are in fact typically very complex in shape. This complexity is important to the manufacture and performance of OSB as it influences forming, screening, blending, formation and ultimately performance of the panel. In a mill setting, strand geometry is defined by average length, width and thickness values as determined through simple caliper measurements and/or screen analyses (Gilson). The application of CCD cameras and digital image analysis techniques to rapidly acquire and analyze complex strand geometry will allow the processing of large amounts of data, thereby creating the potential for statistical process control applications. Aspen (Populus grandidentata) strands were produced from logs subjected to $-6^{\circ} \mathrm{C}(20 \mathrm{~F}), 21^{\circ} \mathrm{C}(70 \mathrm{~F})$ and $60^{\circ} \mathrm{C}(140 \mathrm{~F})$. Grey scale digital images of individual strands were acquired using a CCD camera (1296 x 1016 pixels) under direct lighting. The complexity of strand geometry was characterized by a variety of automated measuring procedures. As a result of this research the feasibility of applying this methodology to study geometrical distributions in strands was established. Area, length and width presented changes in their distributions due to the effect of log temperature. It was found that the irregularity ratio of strands was variable and strongly influenced by log temperature at time of stranding as well as other geometries. Statistical correlation between strands irregularity and log temperature was found ( $\mathrm{p}$-value $<0.001)$.
\end{abstract}

Keywords: Strand Quality, Digital Image Analysis, Non Parametric Statistics, Strand Geometry, Log Temperature

\section{INTRODUCTION}

The anisotropic character of OSB is directly correlated with strand alignment. This relationship has been extensively studied (Geimer, 1975; Shaler, 1991; Mayers, 2001; Zombori, 2001; Barnes, 2002).The influence of variable strand geometries on alignment and packing are known to be important (Dai and Steiner, 1994), but their relationship to optimal process conditions has not been well quantified. Strand overlap and out-of-plane strand orientation (waviness) also influence OSB properties creating weak boundary layers. However their influence is not well quantified.

Typically OSB wood strands exhibit an irregular geometry. This complexity can be quantified both in terms of the variability among and within strands. Variation in thickness, width, length and irregularities along the edges occurs due to handling and machining as well as wood quality variation. However strand geometry is often idealized as being rectangular, smooth and regular in shape (Nishimura et al. 2004). In a mill setting, even for quality control purposes, strand geometry is evaluated basically in terms of an average length, width and thickness values as determined through simple caliper measurements and/or screen analyses (i.e. Gilson test). This approach works fairly well when stable conditions in the logs are present. However, changes in strand geometry can occur due to physical and chemical variations of the wood as a consequence of environmental conditions, reaction wood, natural 
variations in wood quality, and changes in operational behavior of the stranding equipment and knives (FPL, 1999; Negro et al., 2003). Such variation in final strand geometry creates a distribution of geometries which in turn influences the efficiency of the screening and forming processes.

The variation in strand geometries can be best captured and used for decision purposes if a robust statistical description is available. Large sample sizes are beneficial for this purpose; however detailed information on "individual" strand elements in a time effective manner requires an automated or semiautomated procedure.

The use of computer based CCD cameras and image analysis techniques are a powerful and relatively low cost approach to obtain this information. A continuous measurement system may be developed using image analysis, allowing the development of a time-base sampling rather than a weight or count sampling scheme. If accurate, real-time measurements of strand geometry can be obtained it could provide an important input for statistical process control (SPC).

The objectives of this research were:

1) To advance in the development and evaluation of a methodology applicable in an OSB mill to measure and process single strand geometry using digital image analysis;

2) To determine irregularities in strand shape as a consequence of three temperature treatments in the stranding process, and

3) To determine the feasibility of applying this methodology to study the change in single strand geometrical distributions that occurs between the stranding and formation operations in a pilot production line.

\section{MATERIALS AND METHODS}

\section{Strand Generation and Processing}

The study material consisted of three manually debarked Aspen logs of $121.92 \mathrm{~cm}$ (4 feet) in length and ranging from $24.13 \mathrm{~cm}(9.5 \mathrm{in})$ to $25.4 \mathrm{~cm}$ (10 in) in diameter. The logs were cut from the base of one tree selected to be highly cylindrical in shape. Logs were submitted to freezing $\left(-6^{\circ} \mathrm{C} / 20 \mathrm{~F}\right)$, room temperature $\left(21^{\circ} \mathrm{C} / 70 \mathrm{~F}\right)$ and to $60^{\circ} \mathrm{C} / 140 \mathrm{~F}$ soaking from butt log to top log respectively. The strands were produced using a Rotating Carmanah Laboratory Ring Strander (12/48) with target geometry of $0.065 \mathrm{~cm}(0.025 \mathrm{in})$ in thickness, $1.27 \mathrm{~cm}(0.5 \mathrm{in})$ in width and $15.24 \mathrm{~cm}(6 \mathrm{in})$ in length.

The knife cutting angle was $85^{\circ}$ with setback, which was used for the three temperature treatments to attain this target geometry. The strand moisture content (MC) ranged from $89 \%$ for frozen log to $117 \%$ for soaked $\log$. A set of two disposable knives were used to manufacture the strands.

A sensitivity test was conducted using aspen strands in order to determine a minimum sample size to represents the population for image analysis, $4000 \mathrm{~g}$ of dry strands produced from one log were individually measured and from these data 10 sub-samples of different sizes were extracted. To perform the sub-sampling a constant thickness was assumed. The sub-sample was randomly selected by weight considering weight as area multiplied by thickness and wood density. Each sample size was extracted 4 times. From the sub-sample median values for width, length and area were computed and the statistics were compared using a Wilcoxon Sum Rank test. The minimum sample size was determined when the p-value presented a significant level. Weight sampling procedure rather than a count sampling procedure was adopted because picking individual strands would cause a bias on the sample due to handling issues. On the other hand for all production purposes strands are weighed rather than counted. 
From the stranded material, an as a consequence of the previous procedure, a 500g randomly selected sample was taken from each temperature treatment. After that, the strands were dried to a $6 \% \mathrm{MC}$ using a Koch Low Temperature Conveyor Drier and screened using an Acrowood Trillium screener. A new $500 \mathrm{~g}$ sample from each temperature treatment was taken after the screening.

\section{Imaging Process, Measurements and Computations}

The strands were placed in random groups on a rectangular black surface in such a way that arey scale (8-bit) digital image (1296x1016 pixels) of all specimens was acquired. A uniform illumination of $1.2 \times 10^{5}$ Candelas $/ \mathrm{m}^{2}$ was used and all images were kept at a constant scale of $0.0254 \mathrm{~cm} / \mathrm{pixel}$.

Figure 1 shows a diagram of the setup used to capture the images. The CCD camera was connected to a capture interface developed in a LabView environment to a standard PC, and the images stored in a conventional magnetic hard disk drive. Figure 2 shows an example of a group of strands as they were set and recorded. Prior to the computations a Matlab developed subroutine conditioned and filtered the image to adjust contrast. The code also identified and separated the individual strands rotating them if necessary to 0 degrees with respect to the major axis and creating, at the same time, a binary image and a convex hull image of every strand. A binary image is a 2-level image that represents the shape of the image; while a convex-hull image is the smallest convex set of points that contains the points of the binary image or the minimum polygon that contains the region (Barber, 1993). The convex hull image is created and stored to perform area comparison. Through this procedure it is possible to obtain more geometrical information as crack occurrence and determine shape smoothness. Dimensions of the individual strands were measured using a developed MatLab code for digital image analysis (DIA). Width and length measurements of a sub-sample of 200 strands were taken on three different points at each specimen using a Mitutoyo Digital Caliper and compared to the automated DIA measurements of the same sub-sample.

Figure 3 shows a diagram with the processing and measurements that was applied to each strand.

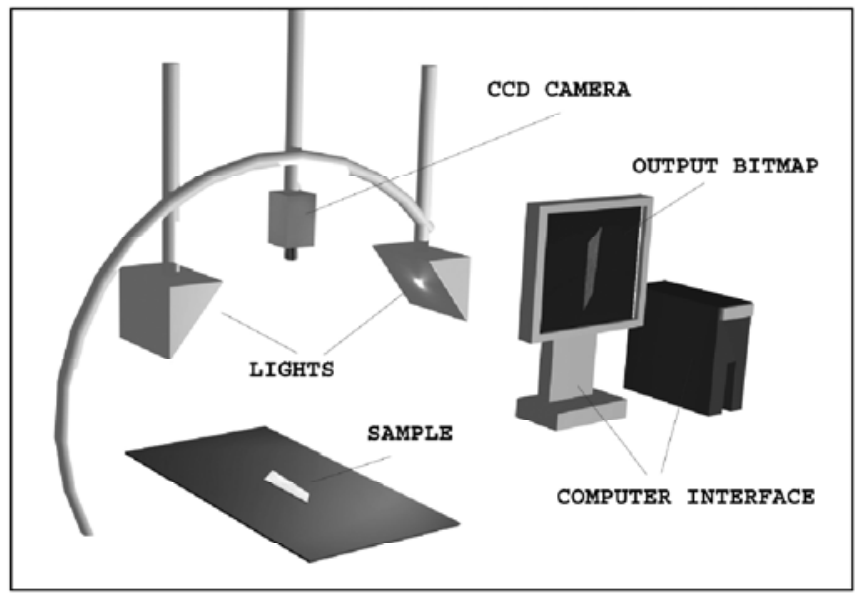

Figure 1: Basic capture setup diagram 


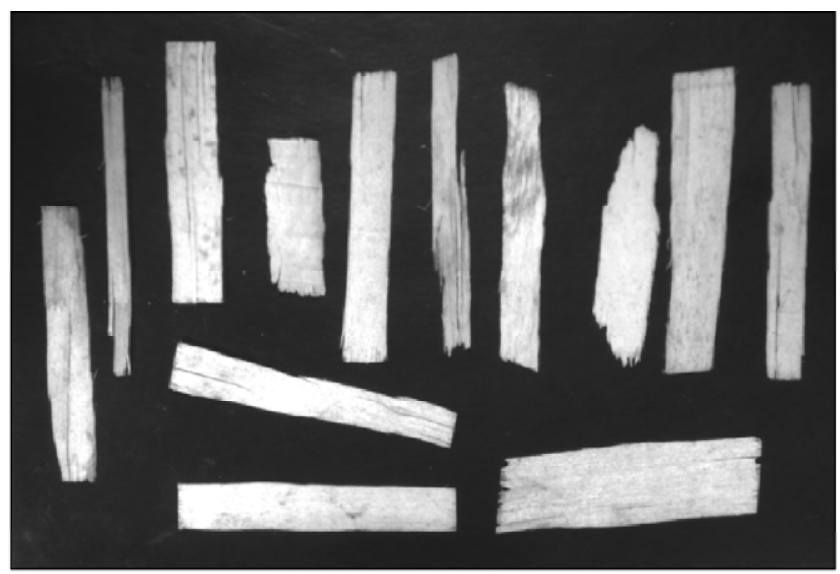

Figure 2: Example image of a group of strands

Since the actual strands exhibit an irregular shape, mean values of width $(W)$ and length $(L)$ were used instead of measuring the strands at a specific point. Transverse and longitudinal pixels averaging rendered average $W$ and $L$ values. Other measures taken were area $(A)$, defined as the sum of pixels in binary image; perimeter $(P)$ defined as the number of pixels in the borderline; and convex hull area $(c H A)$ as previously defined.

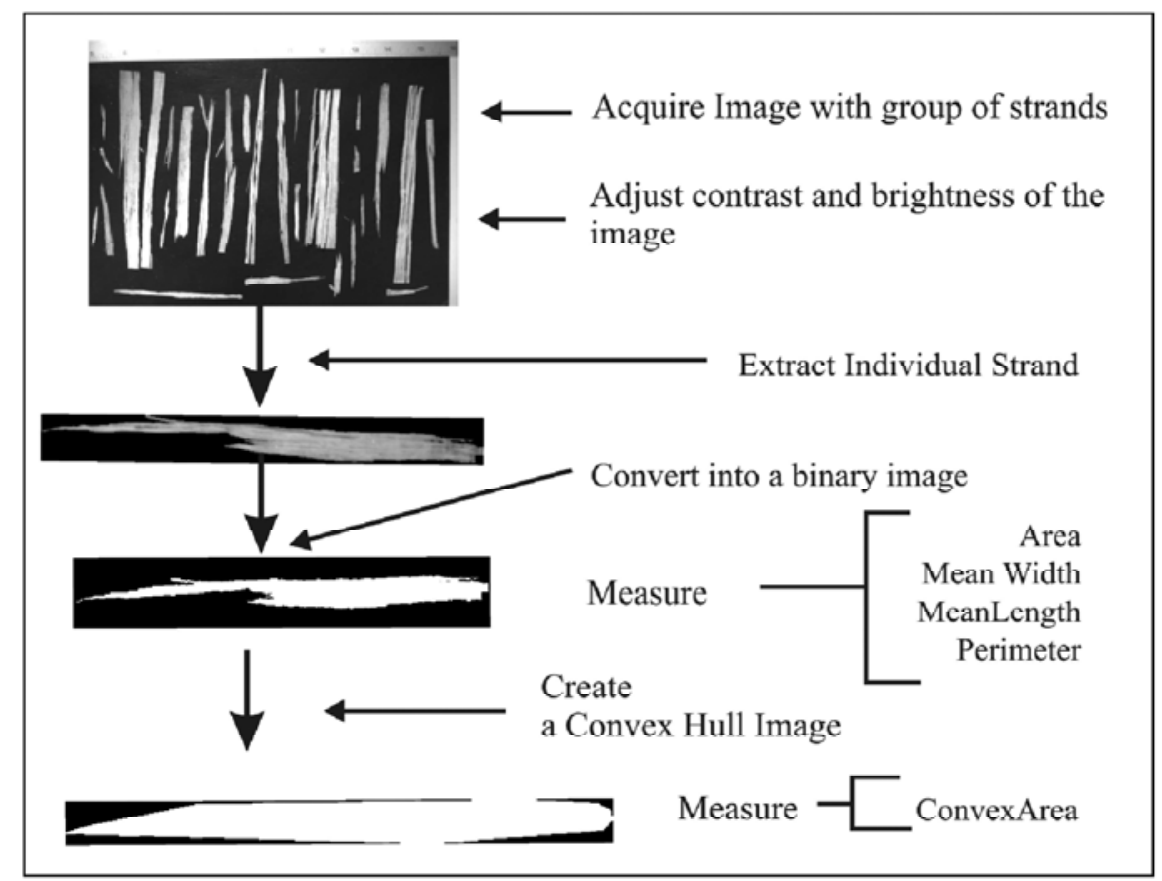

Figure 3: Diagram of Image processing

From the direct measurements on the image the following variables were computed:

1. Shape regularity $(S)$, defined as the proportion of the pixels in the convex hull that are also in the region:

$$
S=\frac{A-c H A}{c H A}
$$


2. Eccentricity $(E c c)$ defined as the ratio of distance between the foci of the ellipse $(d f)$ and major axis length $(l m)$ as shown in Figure 4:

$$
E c c=d f / l m
$$

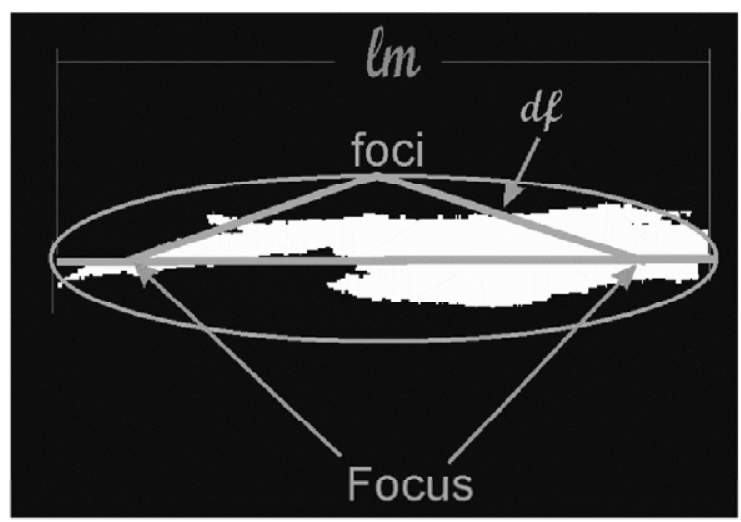

Figure 4: Definition of Strand Eccentricity

3. Slenderness $(S L)$ defined as:

$$
\mathrm{SL}=L / W
$$

4. Rectangularity $(R)$ defined as the proportion of pixels out of the binary region

$$
R=A b / A
$$

where $A b$ is the area of the bounding box. The bounding box is the smallest rectangle that contains the region.

5. Irregularity $(I)$ defined as the absolute value of the ratio between the differences of actual strand perimeter and equivalent ideal rectangle perimeter (in pixels) as in equation (4):

$$
I=\frac{a b s(P-(2 W+2 L))}{(2 W+2 L)}
$$

\section{Statistical Analysis and Experimental Model}

Goodness of fit was performed on all measured and calculated variables at room temperature condition. This procedure was necessary to determine the statistical tools to be used in future research. It was applied to room temperature treatment because it is assumed as the typical processing condition.

The experimental model is designed to study the irregularities on the strand geometry due to log temperature treatments. The simple model can be expressed as

$$
m_{i}=a_{i}+e_{i}
$$


where $m_{i}$ is the percentage of irregularity as defined in eq. (5), $a$ is the temperature treatment and $e_{i}$ is the experimental error.

In evaluating the potential applications for this multiple geometry analysis, 30 bin histograms for all the variables measured and computed were analyzed. Statistical analysis was performed using Statgraphics Centurion XV and R.

\section{RESULTS AND DISCUSSION}

\section{Data acquisition}

The comparison between caliper measurements and digital image measurements is shown in Figure 5. In this graph it is possible to see the accuracy of the measurement provided by the Matlab code $\left(\mathrm{R}^{2}=0.9908\right)$. Small differences are due to the manual selection of the caliper measurement points.

In addition to the accuracy of the measurement, the caliper measurement for this sub-sample required 1.5 minutes/strand of labor for two persons. The cumulative time spent in sampling, image acquiring and processing for DIA was 0.075 minutes/strand for one operator. This time is 40 times faster considering measurements of $W$ and $L$ only.

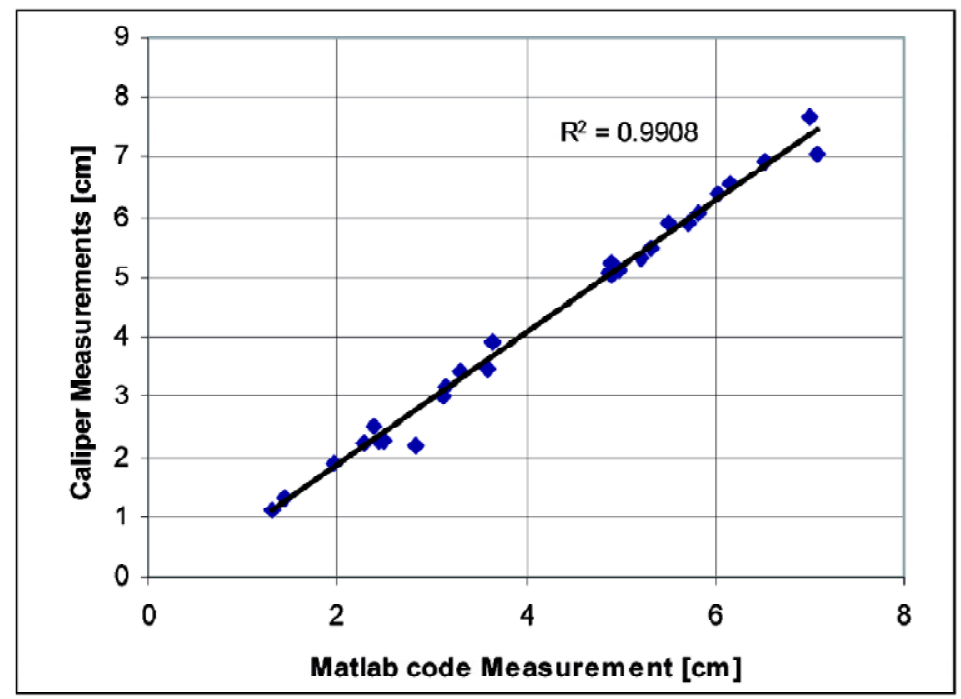

Figure 5: Caliper and Code measurement comparison for mean width

\section{Goodness of fit}

Table 1 summarizes the goodness of fit for different distributions for the variables of this research. A Kolmorogov-Smirnov statistic D was used to determine how well the data fit to the distribution (NIST, 2006). Nine different distributions were tested. For the measured variables $(A, L, P, c H A)$ it is apparent that the best fit distribution is a two-parameter Weibull distribution, although there is not statistical evidence of fitness. $R$ and $I$ fit with normal and Loglogistic distributions respectively. 
Table 1: Summary of Distribution Analysis

\begin{tabular}{|r|c|c|c|c|c|c|c|c|c|}
\hline \multicolumn{8}{|c|}{ Goodness of fit Kolmorogov Smirnov D value for rom temperature log strands } \\
\hline & Weibull & Gamma & Lognormal & Loglogistic & Exponential & Laplace & Logistic & Normal & Uniform \\
\hline A & 0.056 & 0.080 & 0.084 & 0.074 & 0.311 & 0.392 & 0.287 & 0.272 & 0.681 \\
\hline L & 0.089 & 0.087 & 0.112 & 0.104 & 0.180 & 0.276 & 0.197 & 0.180 & 0.368 \\
\hline W & 0.073 & 0.091 & 0.058 & 0.062 & 0.124 & 0.262 & 0.231 & 0.233 & 0.664 \\
\hline P & 0.098 & 0.096 & 0.116 & 0.099 & 0.097 & 0.221 & 0.165 & 0.153 & 0.574 \\
\hline CHA & 0.051 & 0.075 & 0.092 & 0.078 & 0.296 & 0.379 & 0.275 & 0.260 & 0.660 \\
\hline S & & & & & & 0.162 & 0.141 & 0.191 & 0.826 \\
\hline Ecc & 0.227 & 0.299 & 0.306 & 0.302 & 0.516 & 0.363 & 0.290 & 0.285 & 0.736 \\
\hline SL & 0.110 & 0.087 & 0.068 & 0.072 & 0.209 & 0.191 & 0.168 & 0.164 & 0.610 \\
\hline R & $\mathbf{0 . 0 3 1}$ & 0.078 & 0.102 & 0.075 & 0.313 & 0.058 & 0.037 & $\mathbf{0 . 0 3 1}$ & 0.146 \\
\hline I & 0.061 & 0.059 & 0.068 & $\mathbf{0 . 0 2 9}$ & 0.099 & 0.159 & 0.134 & 0.168 & 0.645 \\
\hline
\end{tabular}

Relative histograms for $A, L$ and $W$ are shown in frozen, room and soaked conditions in Figure 6 . In this figure it is possible to see the changes in the geometrical distributions for strands in response to the temperature treatments applied to the logs and also, it is possible to visually confirm that the distributions do not fit normal distributions. The same behavior was detected in all the other measured and computed variables.

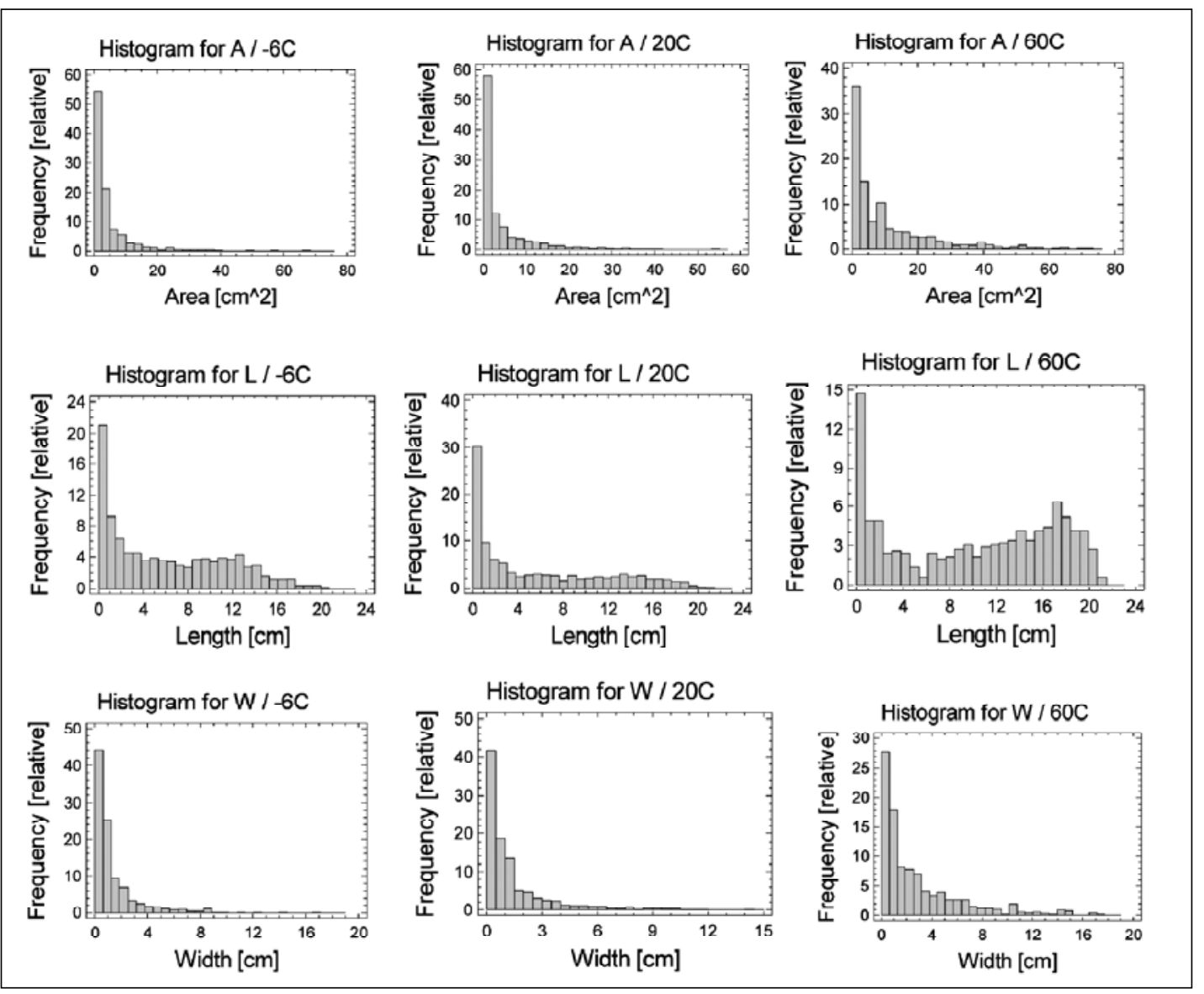

Figure 6: Actual distributions for A, L and $\mathrm{W}$ and three temperatures 


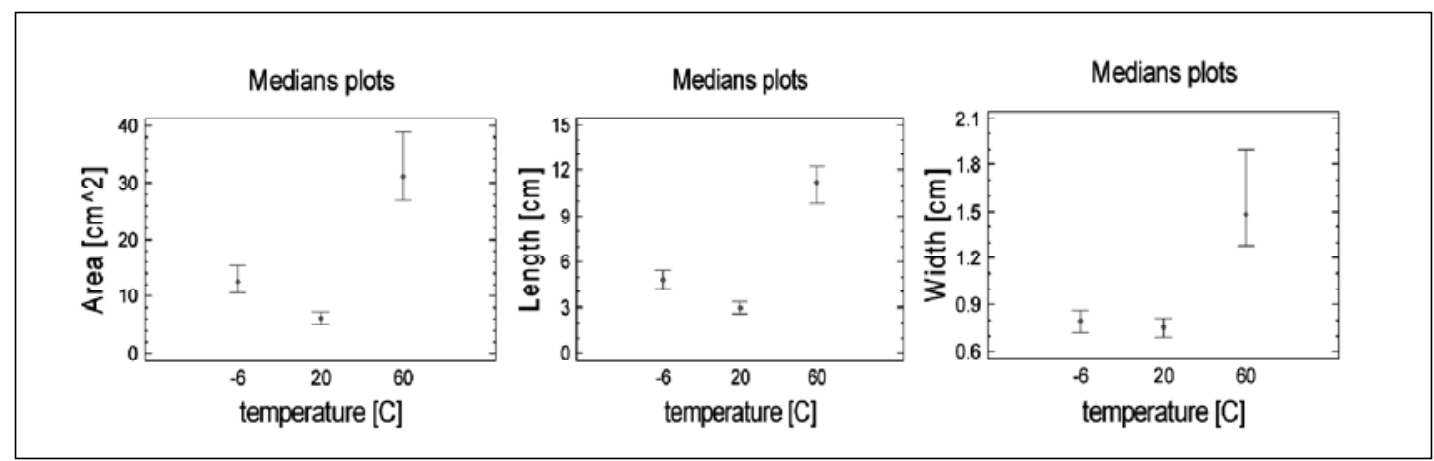

Figure 7: Medians comparison for A, L and W

The influence of soaking on median area $(A)$, length $(L)$ and width $(W)$ of strands is shown in Figure 7. In this figure it is noticeable that there is no difference in $W$ between frozen and room temperature treatments; and the effect tends to be inverse to the expected in $A$ and $L$. This phenomenon can be due to the unusual low MC of the frozen log, which might affect the freezing. Since mean width, length and area are often considered as response variables when using image analysis, a special caveat has to be taken in account to properly analyze the results.

Figure 8 presents the distribution of $I$ for the three log temperatures. A simple regression to determine the correlation between $I$ and temperature was conducted as shown in Table 2. According with the ANOVA table, there is a statistically significant relationship between $I$ and log temperature (Figure 9).

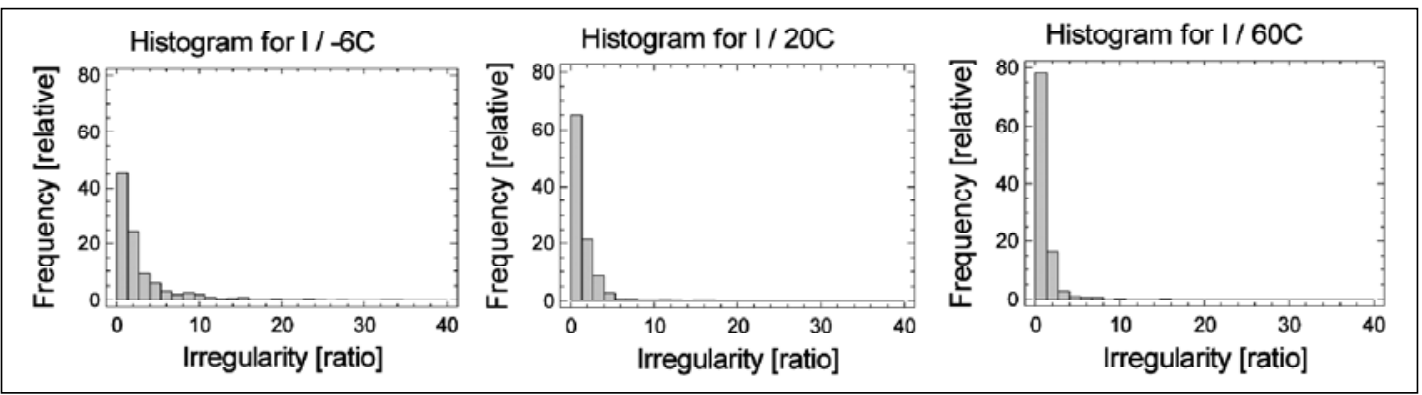

Figure 8: Distributions for I and log temperature

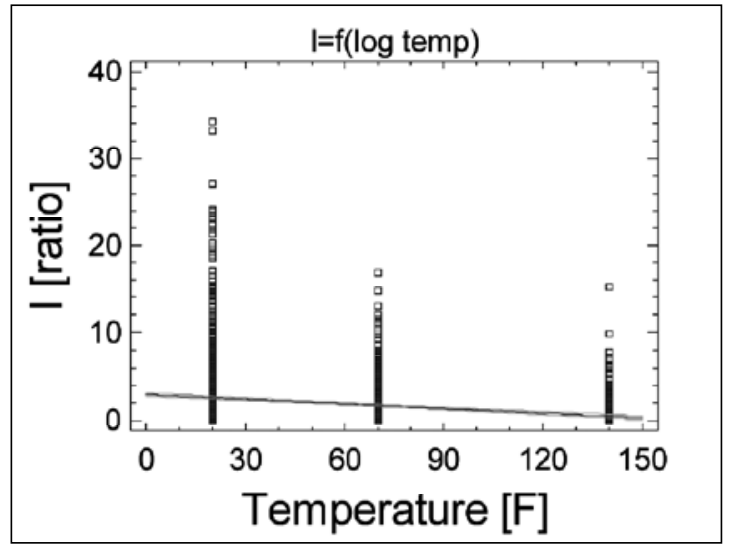

Figure 9: Correlation between Irregularity and Temperature 
Figure 10 shows the distributions for $R$ upon the different conditions. As stated earlier, this is the only variable that behaves statistically normal for room conditions. Significant relationship of $R$ and $\log$ temperature was found as well $(\mathrm{F}=314.19$ with a $\mathrm{p}<0.01$ in the ANOVA).

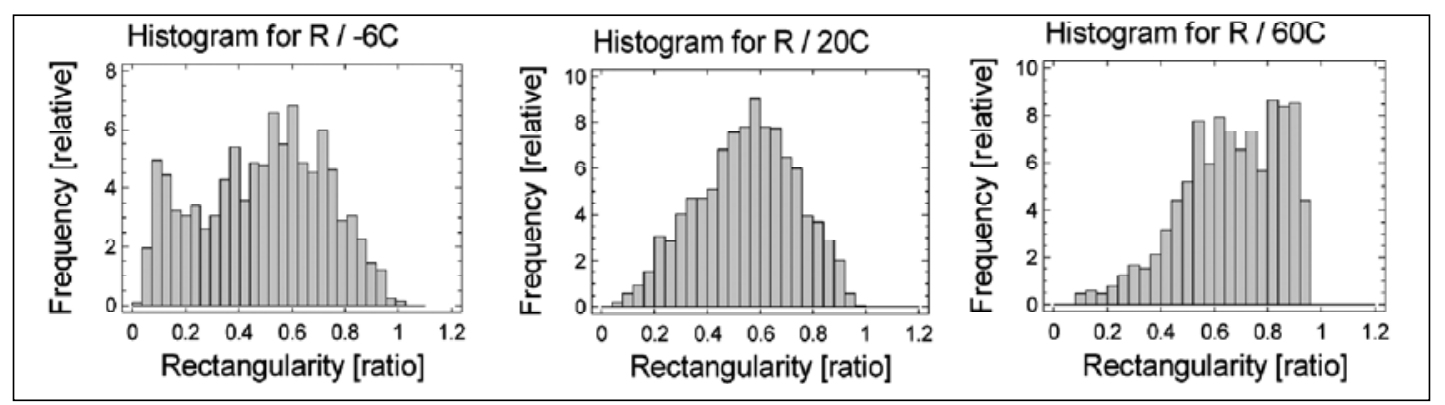

Figure 10: Distributions for $R$ and log temperature

Table 2: Linear regression on I v/s log temperature

Linear model: $\mathrm{Y}=\mathrm{a}+\mathrm{b} * \mathrm{X}$

\begin{tabular}{|l|l|l|l|l|}
\hline & Least Squares & Standard & $T$ & \\
\hline Parameter & Estimate & Error & Statistic & $\begin{array}{l}P \text { - } \\
\text { Value }\end{array}$ \\
\hline Intercept & 2.98119 & 0.0821298 & 36.2985 & $<0.001$ \\
\hline Slope & -0.0175736 & 0.00105427 & - & $<0.001$ \\
\hline
\end{tabular}

Analysis of Variance

\begin{tabular}{|l|l|l|l|l|l|}
\hline Source & $\begin{array}{l}\text { Sum of } \\
\text { Squares }\end{array}$ & Df & $\begin{array}{l}\text { Mean } \\
\text { Square }\end{array}$ & $\begin{array}{l}\text { F- } \\
\text { Ratio }\end{array}$ & P-Value \\
\hline Model & 1885.36 & 1 & 1885.36 & 277.85 & $<0.001$ \\
\hline Residual & 25465.7 & 3753 & 6.78543 & & \\
\hline $\begin{array}{l}\text { Total } \\
\text { (Corr.) }\end{array}$ & 27351.1 & 3754 & & & \\
\hline
\end{tabular}

The change in geometry and specifically in irregularity and rectangularity due to the temperature treatment can be explained as changes in wood reducing stiffness and increasing plasticization of wood matrix (lignin) as log temperature increases. On the other hand, in frozen conditions wood behaves more brittle; the stranding knives cut and break fibers following random weak planes existing in the frozen composite rather than following the knife edge. This fact promotes the occurrence of larger fines fractions and it may affect screening and alignment processes by the creation of strand clusters. 


\section{CONCLUSIONS}

Digital image analysis is a useful technique to process high amount of data and quickly and accurately determine geometrical distributions. The potential use of this methodology for on line measurements is noticeable. Multiple variable and highly detailed measurements can be done with this setup using available and inexpensive equipment.

Log temperature has an important effect in the geometry of the strands. As temperature increases from $20^{\circ} \mathrm{C}$ to $60^{\circ} \mathrm{C}$, larger and less irregular strands are produced. However, further research is necessary to determine the cause of reduction in geometries from $-6^{\circ} \mathrm{C}$ to $20^{\circ} \mathrm{C}$. Irregularity, as defined in this paper, is the geometrical property that exhibited the strongest statistically significant effect of $\log$ temperature on stranding.

Non-parametric statistics must be applied to study the geometrical distribution of strands. Mood's Median comparison, Kruskal-Wallis, Kolmorogov-Smirnov or other statistical tools must be applied to study this type of data. A further study will be necessary to develop optimum statistical tools to analyze this type of data and develop protocols to evaluate the results on a time-base sample scheme for continuous process.

Additional research is necessary to study the relationship between the irregularities on strands geometry and the effectivenessof screening and on mat formation process respect to the strand alignment.

\section{ACKNOWLEDGEMENTS}

This work was supported in part by USDA/CSREES Special Grant Award F 2003-34158-14006

The authors wish to acknowledge Russell Edgar Wood Composite Manager and Jon Hill Scientific Technician from the AEWC Center of the University of Maine for their valuable help in this research.

\section{REFERENCES}

Barber, C. B.; Dobkin, D. P.; Huhdanpaa, H. 1993. The Quickhull Algorithm for convex hull. Tech. Rep. GCG53, The Geometry Center, Univ. of Minnesota, Minneapolis, Minn.

Barnes, D. 2001. A model of the effect of strand length and strand thickness on the strength properties of oriented wood composites. Forest Products Journal 51(2) 36-46.

Barnes D.; Ens,J. E. 1988. Strand Orientation Sensing, United States Patent 5764788, 6-9-1988.

Chen, S. http://www.arc.ab.ca/Index.aspx/ARC/4367, 2005-2006 Alberta Research Council.

Dai, C.; Stainer, P. R. 1994. Spatial structure of wood composites in relation to processing and performance characteristics Part 3. Modeling the formation of multi-layered random flake mats, Wood Science and Technology, Volume 28, Number 3, March 1994, pp: 229 - 239

El-Sombaty, Y.; Ismail, M.A. 2003. Matching Occluded Objects Invariant to Rotations, Translations, Reflections, and Scale Changes. In Proceedings of the 13th Scandinavian Conference on Image Analysis, SCIA 2003, July 2003. pp 836-843. 
Forest Products Laboratory. 1999. Wood Handbook - Wood as an engineering material. Gen. Tech. Rep. FPL-GTR-113. Madison, WI. U.S. Department of Agriculture, Forest Service, Forest Products Laboratory. 1999. 463 p.

Geimer, R. L.; Montrey, H.M.; Lehmann, W. F. 1975. Effects of layer characteristics on the properties of three-layer particleboards. Forest Products Journal Vol 25 No. 3. 1975. pp. 19-29.

Hu, C.; Afzal, M., 2005. Automatic measurement of wood surface roughness by laser imaging. Forest Product Journal 55 (12):158-163.

Mayers, K. L.; 2001. Impact of strand geometry and orientation on mechanical properties of strand composites, Thesis Ms. Sc. In Civil Engineering, Washington State University. 2001.

Negro, M. J.; Manzanares, P.; Oliva, J. M.; Ballesteros, I.; Ballesteros, M. 2003. Changes in various physical/chemical parameters of Pinus pinaster wood after steam explosion pretreatment, Biomass \& bioenergy 25(3): 301-308.

Nishimura, T.; Amin, J.; Ansell, M. P. 2004. Image analysis and bending properties of model OSB panels as a function of strand distribution, shape and size. Wood Science and Technology (38): 297-309.

Quinn, G. P.; Keough, M. 2002. Experimental design and data analysis for biologists. Publisher New York; Cambridge University Press.

Sladoje N.; Nystrom, I.; Saha, P. K. 2003. Measuring perimeter and area in low resolution images using fuzzy approach. In Proceedings of the 13th Scandinavian Conference on Image Analysis, SCIA 2003, July 2003. pp 853-860.

Shaler, S.M. 1991. Comparing two measures of flake alignment. Wood Science and Technology 26(1): 53-61.

Zombori, B.G. 2001. Modeling the transient effects during the hot-pressing of wood-based composites. Ph.D. Dissertation. Virginia Polytechnic Institute. 2001. 
